\title{
Acute Epidural Hematoma after Ventriculoperitoneal Shunt Insertion: A Case Report
}

\author{
Hamid Etemadrezaie ${ }^{1}$, Samira Zabihian ${ }^{1}$, Humain Baharvahdat ${ }^{1}$, Babak Ganjeifar ${ }^{1 *}$ \\ ${ }^{1}$ MD, Assistant Professor of Neurosurgery, Department of Neurosurgery, Mashhad University of Medical Sciences (MUMS), Mashhad, Iran \\ * Corresponding Author Address: Ghaem Hospital, Department of Neurosurgery, Mashhad University of Medical Sciences (MUMS), Mashhad, \\ Iran. Email: b_ganjeifar@yahoo.com and ganjeifarb@mums.ac.ir. Tel: +98(513)8012613, Fax: +98(513)8413493 \\ Article Type: Case Report \\ Received: December 4, 2015, Last revised: December 8, 2015, Accepted: December 21, 2015
}

\section{Abstract}

Background \& Importance: Acute epidural hematoma is a very rare complication of ventriculoperitoneal shunt insertion. The insertion of a ventriculoperitoneal shunt can cause sudden decompression of the brain, subsequent to which epidural hematoma occurs due to CSF drainage. To our knowledge, there are only a few cases of acute epidural hematoma in the literature which required acute evacuation.

Case Presentation: In this report, we present a case of epidural hematoma close to ventriculoperitoneral shunt insertion site in a 30-year-old man after failure of endoscopic surgery for opening of the wall of a suprasellar arachnoid cyst. Secondary to communication between cyst and ventricles and clinical symptoms and sings, the patient underwent the shunt insertion. The patient became comatose two hours following the insertion of the shunt, developing a voluminous right temporo-parietal epidural hematoma that had to be evacuated immediately. Here, we intend to discuss both the pathophysiology and treatment.

Conclusion: Development of epidural hematoma after ventriculoperitoneal shunt surgery is a devastating complication. Dehisensce formation between the skull and dura matter, which may be facilitated by lax adhesion between the two, is a common underlying pathology. We recommend a close post-surgical observation for immediate diagnosis and reoperation of this event.

Keywords: Acute; Epidural; Hematoma; Ventriculoperitoneal shunt

Please cite this paper as: Etemadrezaie H, Zabihian S, Baharvahdat H, Ganjeifar B. Acute Epidural Hematoma after Ventriculoperitoneal Shunt Insertion: A Case Report. Iran. J. Neurosurg. 2015;1(3):30-32.

\section{Introduction}

Epidural hematoma in the operative site is an uncommon morbid complication seen in neurosurgery. Epidural hematoma after shunt insertion was reported as isolated cases in literatures (1-5).

This case is reported with the intention of comparing and contrasting the risk factors and mechanisms with similar ones documented before. It is also intended to promote insight into one of the rare complications following a common procedure, which can easily been prevented from or, in case of occurrence, detected and treated accordingly.

\section{Case Presentation}

A 30-year-old man was admitted to our ward with presenting complaint of morning headache, nausea and blurred vision. Endoscopic surgery has been carried out twice, 55 and 34 days before, respectively, with the aim of opening the wall of suprasellar arachnoid cyst. Nevertheless, signs and symptomsnausea, vomiting and blurred vision, failed to resolve.

Given the hydrocephalus and communication between the cyst and ventricular system in the MRI (Figure 1) and CTventriculography (Figure 2), persistent papilledema and ataxia, we decided to treat the patient by applying a medium-pressure ventriculoperitoneal shunt.

A ventriculoperitoneal shunt with a medium pressure flow control valve was inserted through a right temporooccipital burr-hole.

Two hours after the end of the surgery, the patient's consciousness decreased (GCS=13), developing left hemiplegia.

An immediate brain CT scanning revealed a voluminous right temporoparietal epidural hematoma (with about 75 milliliter volume) (Figure 3).

The patient was taken to the operating room where a large temporoparietal craniotomy was performed following the ligation of the distal catheter in the upper chest. The posterior border of the bony flap was the temporooccipital burr-hole. Origin of the hematoma was terminal branch of the posterior division of the middle meningeal artery.

Due to a large epidural dead space after the removal of epidural hematoma, we had to infuse more than 100 milliliter of normal 


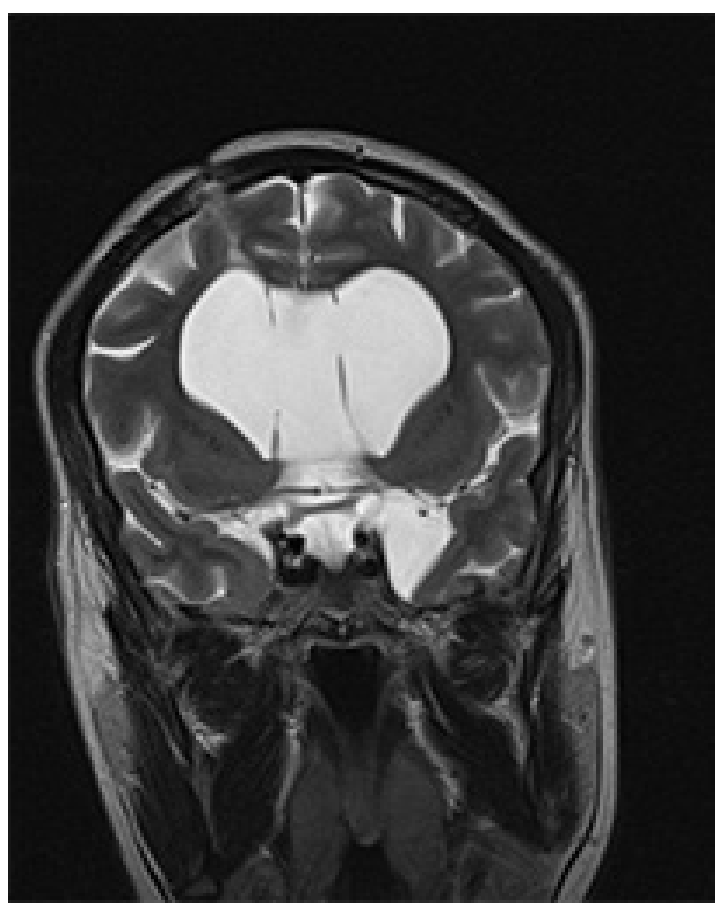

Figure 1. Coronal T2-weighted MRI after Endoscopic Surgery

saline into the ventricular catheter to expand the dura matter. Postoperative CT showed the complete removal of the hematoma (Figure 4). Pre and postoperative coagulation profile was normal. Distal catheter was open after one month.

The patient was alert, but hemiparetic (upper limb 2/5 and lower limb 3/5) on discharge, with the complete resolution of the headache. Papilledema, however, took longer to resolve during follow-up, whereas hemiparesis persisted for more than four months with complete recovery after that.

\section{Discussion}

A drastic decline in the ICP may well cause subdural collection following ventricular drainage, particularly following chronic hydrocephalus in those with craniocerebral discrepancy (6).

Nevertheless, epidural hematomas rarely occur in such cases owing to the adherence of the dura to the skull (7).

Even so, it occurs in younger patients $(1-3,6-8)$ at anatomical sites in which pathology is more likely to be detected (parietal and frontal regions) (4).

Another explanation is that the dura arachnoid attachments are more considerable than skull dura adhesion in certain cases, giving rise to epidural hematoma $(\mathrm{EDH})$ rather than subdural hematoma (3).

This can often be seen in infants whose dura matter is loosely attached to the inner cranium (9).

Detachment of dura matter from inner table of the skull may initially cause dural and diploic veins bleeding, thereby widening the gap between the dura matter and bony arterial cannels, which, in turn, causes dural arteries tearing (7).

Another theory state that what has led to the shrinkage and thus segregation of the dura from the skull regards excessive coagulation of the dura at the burr hole prior to incision. Valveregulated shunts, however, diminish subdural collections following drainage, so do the same in cases of epidural hematoma (7).

The incidence of epidural hematoma after ventricular shunt operation was $5.6 \%$ in adults and $0 \%$ in children before the

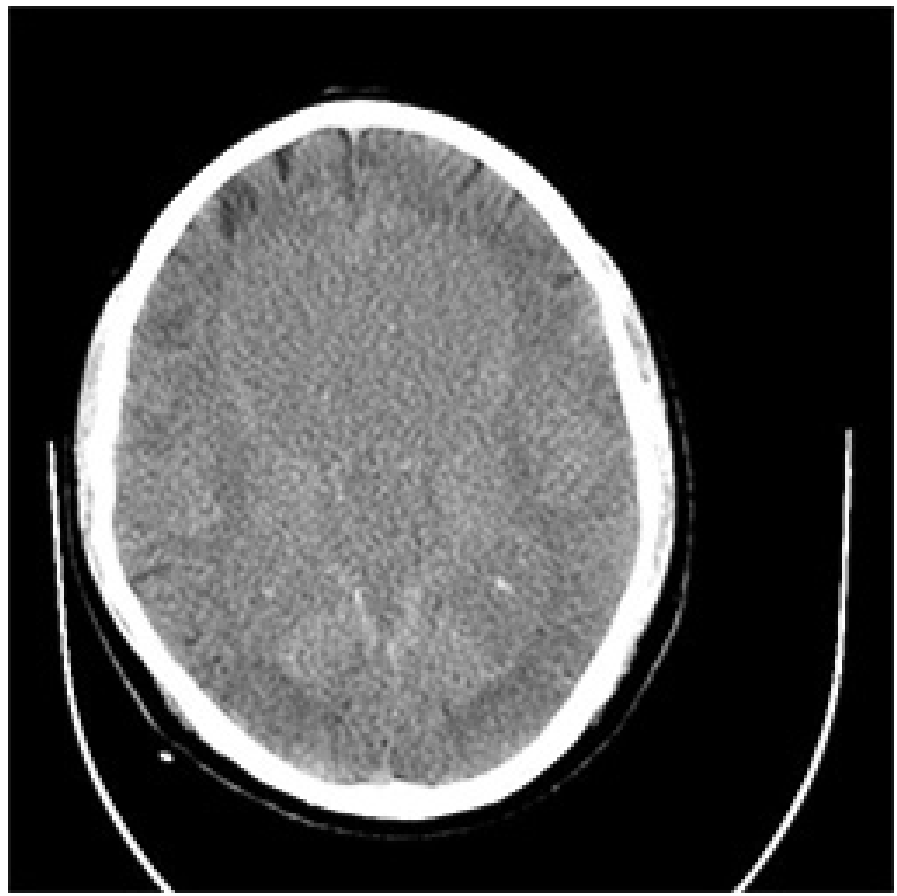

Figure 2. Axial CT-ventriculography before Shunt Insertion

advent of valve-regulated shunt (1).

In our knowledge, 15 acute and 7 chronic cases of epidural hematomas were reported after valve-regulated shunt placement in the literature $(2,3,5,10-12)$.

Epidural hematoma often becomes symptomatic soon after a neurosurgical operation, whereas diagnosis can be procrastinated in a case of an efficient shunt which helps reduce intraventricular pressure and volume. As in this case, it may merely present as headache, or seizures in delayed ones (7).

Hematomas were unrelated to the burr-hole sites in most of the acute epidural hematoma cases due to a ventriculoperitoneal shunt, occurring more frequently in the frontal region $(1,4)$; this can be explained as there are stronger attachments to bone in the posterior rather than anterior cranium (5). In our patient, the hematoma was related to the burr-hole site.

We believe that the forced introduction of the ventricular catheter through a narrow dura matter hole coupled with volumetric drainage of CSF in our patient who had been chronically high ICP pressure had led to the detachment of dura matter and subsequently huge epidural hemorrhage.

There are two distinct approaches for treament of epidural hematomas in these cases; conservative and surgical, the choice for or against of which depend on clinical setting, age, time of bleeding, size on CT-scan, extent and thickness of the hematoma and the presence of midline shifting on CT. Any rapid accumulation in children and younger adults must be treated surgically.

This condition can be prevented with bearing of the following precautions in mind.

First and foremost, ventricular catheter must be applied only with minimal CSF spillage. Besides, pressure valves must be selected accordingly (medium or high pressure valves have priority). The patient should return to upright position slowly and gradually, while follow up CT-scan is routinely performed. The use of any flow rate-limiting system including an antisiphon device will be of great benefit (7).

We also believe that creating an opening with an appropriate 


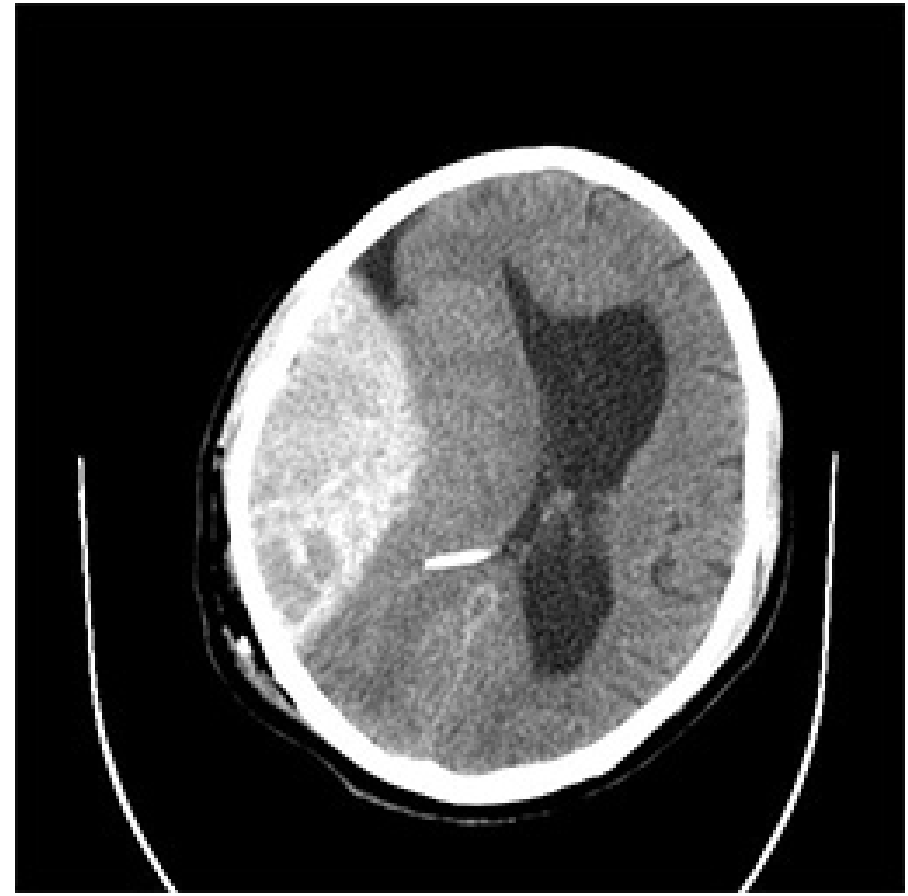

Figure 3. . Axial CT Three Hours after Shunt Insertion

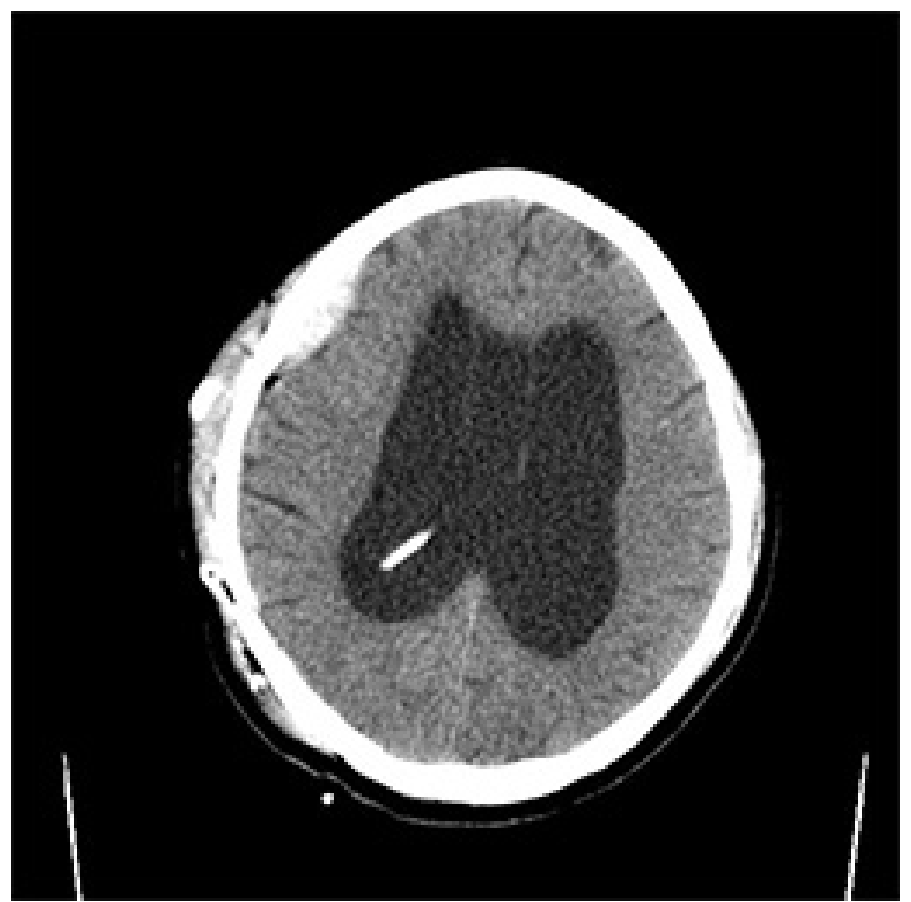

Figure 4. . Axial CT after Removal of EDH

size in the dura matter for the ventricular catheter insertion prevents the dehiscence of the dura matter from the skull.

\section{Conclusion}

Development of epidural hematoma after ventriculoperitoneal shunt surgery is a devastating complication. Dehisensce formation between the skull and dura matter, which may be facilitated by lax adhesion between the two, is a common underlying pathology. We recommend a close post-surgical observation for immediate diagnosis and re-operation of this event.

\section{Funding}

None.

\section{Conflicts of Interest}

The authors declare that they have no conflicts of interest.

\section{References}

1. Erdogan B, Sen O, Bal N, Cekinmez M, Altinors N. Rapidly calcifying and ossifying epidural hematoma. Pediatric neurosurgery. 2003;39(4):208-211.

2.Kalia KK, Swift DM, Panz D. Multiple epidural hematomas following ventriculoperitoneal shunt. Pediatric neurosurgery. 1993;19(2):78-80.

3. Mathuriya SN, Kak VK, Banerjee AK. Ossified epidural hematomas: Report of two cases. Clinical neurology and neurosurgery. 1989; 91(3):269-272

4. Sena JC, Grazian N. Hematoma epidural como complicação de uma drenagem ventricular externa. Arq Bras Neurocirurg 1992; 11(3):153-158.

5. Tjan TG, Aarts NJM. Bifrontal hematoma after shunt operation and posterior fossa exploration. Report of a case with survival. Neuroradiology.1980; 19(1):51-53.

6. Seyithanog lu H, Karag Oz Guzey F, Emel E, Ozkan N, Aycan A. Chronic Ossified Epidural Hematoma After Ventriculoperitoneal Shunt Insertion: A Case Report. Turkish Neurosurgery. 2010; 20(4) 519-523.

7.Jain SK, Sundar IV, Sharma V, Arota R, Prasanna KL.Chronic ossified extradural hematoma on the opposite side of the ventriculoperitoneal shunt procedure: A rare case report. Saudi Journal for Health Sciences. 2012; 1(3):159-161.

8. Schorstein J. Fatal intracranial venous haematoma following ventricular drainage. Journal of neurology and psychiatry. 1942; 5(3-4):142

9. Sengputa RP, Hankinson J. Extradural hemorrahge: a hazard of ventricular drainage. Journal of Neurology, Neurosurgery \& Psychiatry.1972; 35(3):297303

10.Chauvet D, Sichez JP, Boch AL.Early epidural hematoma after CSF shunt for obstructive hydrocephalus. Neuro-Chirurgie. 2009; 55(3):350-3.

11.Pereira C U, De Souza Porto M W, De Holanda R R, De Andrade W T.Epidural Hematoma After Ventriculoperitoneal Shunt Surgery.Report of two cases. Arquivos de neuro-psiquiatria. (3B):629-632,1998

12.Harkness W.Contralateral extradural haematoma after ventriculoperitoneal shunt insertion. Journal of the Royal Society of Medicine. 1999; 92(10):547. 